\title{
Etude du Spectre Pour Certains Noyaux sur un Arbre.
}

\author{
Ferdaous Kellil(*) - Guy Rousseau(**)
}

ABSTRACT - We study in this paper the spectrum of some kernels acting on a locally finite tree, in particular those associated to an anisotropic random walk on the tree with jumps of length 0,1 or 2 . Such a kernel is a function $R$ on $S \times S$ where $S$ is the set of vertices of the tree, it acts on $\ell^{r}(S)$. We always assume the kernel $R$ to be invariant under the action of a group $\Lambda$ of automorphisms almost transitive on $S$. This work generalizes results of A. Figà Talamanca and T. Steger who deal with homogeneous trees and a fixed group $\Lambda$, simply transitive on $S$; it shows the diversity of the spectrum depending on the invariance group.

RÉSUMÉ - L'objectif de cet article est l'étude du spectre de certains noyaux agissant sur un arbre localement fini, en particulier ceux associés à une marche aléatoire sur l'arbre à sauts de longueur 0,1 ou 2 . Un tel noyau est une fonction $R \operatorname{sur} S \times S$ où $S$ désigne l'ensemble des sommets de l'arbre; il agit sur $\ell^{r}(S)$. Nous supposerons toujours le noyau $R$ invariant par un groupe $\Lambda$ d'automorphismes presque transitif sur l'arbre. Ce travail généralise des résultats de A. Figà Talamanca and T. Steger qui étudient le cas des arbres homogènes et d'un groupe $\Lambda$ fixé, simplement transitif sur $S$; il fait ressortir la diversité du spectre selon la nature du groupe d'invariance.

\section{Introduction.}

A. Figà-Talamanca, T. Steger ([St] ou [Ft-S]) et K. Aomoto [A] ont les premiers étudié la résolvante d'un noyau anisotrope sur un arbre. Les résultats de [Ft-S] sur le spectre sont obtenus pour un noyau symétrique sur

(*) Indirizzo dell'A.: Faculté des sciences de Monastir. Département de Mathématiques, 5000 Monastir, Tunisie.

E-mail: kellilferdaous@yahoo.fr

(**) Indirizzo dell'A.: Institut Elie Cartan. Unité mixte de Recherche 7502. Nancy-Université, CNRS, INRIA. B.P (239), 54506 Vandoeuvre Lès Nancy, France.

E-mail: rousseau@iecn.u-nancy.fr 
un arbre homogène, invariant par un groupe particulier simplement transitif sur les sommets. Nous continuons cette étude pour un arbre localement fini plus général, et faisons en particulier apparaitre des phénomènes nouveaux liés aux différentes possibilités pour le groupe d'invariance du noyau considéré.

On se donne un arbre localement fini $X$, et la décomposition de son ensemble $S$ de sommets en deux parties $S^{\prime}$ et $S^{\prime \prime}$ selon la parité de la distance. Pour $s \in S$ on note $\Sigma(s, n)$ la sphère de centre $s$ et de rayon $n$ formée des sommets à distance $n$ de $s$.

On considère un sous groupe $\Lambda$ de Aut $(X)$ qui stabilise $S^{\prime}$ et $S^{\prime \prime}$ et qui est transitif sur $S^{\prime}$. Alors tout sommet de $S^{\prime}$ a un nombre fixe $q+1$ de voisins. On dit que l'arbre est semi-homogène (resp. homogène) si tout sommet de $S^{\prime \prime}$ a un nombre fixe $\ell+1$ de voisins (resp. et si $q=\ell$ ).

Un noyau complexe sur l'arbre (ou sur $S^{\prime}$ ) est une application $R$ de $S \times S$ (ou $S^{\prime} \times S^{\prime}$ ) dans C. La formule $R * f(s)=\sum_{t \in S} R(s, t) f(t)$ permet de faire opérer ce noyau sur certaines classes de fonctions sur $S$ (ou $S^{\prime}$ ).

Le noyau $R$ est supposé invariant par 1 ; i.e. $R(\lambda(s), \lambda(t))=R(s, t)$, $\forall s, t \in S$ et $\forall \lambda \in \Lambda$. Si $R$ est invariant par un groupe $\Lambda$ simplement transitif, (cf. [Ft-S]) on peut identifier $R$ à une fonction sur $\Lambda$ qui opère par convolution.

Pour étudier le spectre d'un tel noyau on suppose soit $R(s, t)=0$ pour $d(s, t) \neq 1$ (noyau à sauts de longueur 1 ) soit $R(s, t)=0$ pour $d(s, t) \neq 0$ ou 2 (noyau à sauts de longueur 0 ou 2); ce dernier cas nous permet d'étudier le carré $R^{2}(s, t)=\sum_{u} R(s, u) R(u, t)$ d'un noyau du type précédent.

Pour montrer que certains complexes sont hors du spectre, on utilise une généralisation du théorème de Haagerup prouvée en [K-R.1] sous une condition technique $(U)$ sur $\Lambda$ toujours vérifiée si $\Lambda$ est discret. Plus précisément dans le cas où $\Lambda$ est fermé dans $\operatorname{Aut}(X)$ la condition $(U)$ est équivalente à l'unimodularité de $\Lambda$, (cf. [B.K; 3.1]).

Pour un tel noyau, le spectre formel (sans condition de convergence) n'a pas grande signification. En effet on montre au debut du premier paragraphe comment construire des fonctions $z$-harmoniques sur $S$ à partir de n'importe quelle fonction harmonique. On généralise ainsi un résultat de $\mathrm{J}$. M. Cohen et F. Colonna [C-C].

On s'intéresse dans la suite du paragraphe 1 au spectre $\ell^{r},(1 \leq r \leq 2)$ d'un noyau $R$ sur $S$ à sauts de longueur 1 invariant par un groupe $\Lambda$ transitif sur $S^{\prime}$. On suppose connexe le complémentaire du spectre $\ell^{r}$ de $R$, ce qui permet d'affirmer que l'expression générique de la résolvante (déterminée en [K-R.2]) est valable partout en dehors du spectre. Si 0 est hors du spectre $\ell^{r}$ de $R$, on montre alors en 1.7 que $\Lambda$ stabilise un ensemble d'arêtes dites 
spéciales (tel que tout sommet de $S$ soit dans une et une seule arête spéciale), l'arbre est semi-homogène et le noyau $R$ est fortement anisotrope : $\left|R\left(s, t_{1}\right)\right|^{r}>\sum_{i=2}^{n}\left|R\left(s, t_{i}\right)\right|^{r}$ si $s \in S, \Sigma(s, 1)=\left\{t_{1}, \cdots, t_{n}\right\}$ et $\left\{s, t_{1}\right\}$ spéciale.

Sous la condition $(U)$ on a en fait équivalence et l'arbre est alors forcément homogène. On généralise ainsi des résultats de $\mathrm{A}$. Figà Talamanca et Tim Steger [Ft-S] qui traitent le cas d'un noyau $R$ symétrique et d'un groupe d'invariance $\Lambda$ particulier.

Au second paragraphe on étudie les mêmes questions pour un noyau $R^{\prime}$ sur $S^{\prime}$ à sauts de longueur 0 ou 2 , et avec un groupe d'invariance $\Lambda$ (transitif sur $S^{\prime}$ ) tel que le fixateur de tout sommet $s \in S^{\prime \prime}$ est doublement transitif sur l'ensemble de ses voisins. Sous les mêmes conditions $(U)$ et de connexité du complémentaire du spectre, on obtient au théorème 2.6 une condition nécessaire et suffisante pour que $\gamma_{0}\left(\gamma_{0} \neq 0\right)$ vérifiant $R_{\gamma_{0}}^{\prime}(s, s)=0$ soit en dehors du spectre $\ell^{r}$ de $R^{\prime}$. On explique aussi en 2.13 un procédé de détermination générale du spectre $\ell^{2}$ de $R^{\prime}$.

Soit $R$ un noyau à valeurs toutes réelles ou toutes imaginaires pures, à sauts de longueur 1 et invariant par le même groupe $\Lambda$ qu'à l'alinéa précédent. Alors la restriction $R^{\prime}$ de $R^{2}$ à $S^{\prime}$ vérifie les conditions précédentes (pour $r=2$ ) et on en déduit des précisions sur le spectre $\ell^{2}$ de ce noyau; en particulier 0 est toujours dans le spectre $\ell^{2}$ et même le spectre $\ell^{r}, 1 \leq r \leq 2$ sous l'hypothèse de connexité du complémentaire du spectre $\ell^{r}$.

\section{Etude du spectre pour certains noyaux sur $S$.}

1.1. Un premier résultat consiste à généraliser les travaux de J. M. Cohen et F. Colonna [C-C] qui concernent les arbres homogènes avec un noyau isotrope à sauts de longueur 1 :

Soit $R$ un noyau à sauts de longueur 1 sur $S \times S$, on dit qu'une fonction $f$ sur $S$ est harmonique (resp. $z$-harmonique pour $z \in \mathbb{C}$ ) si $\Delta f=(R-I) * f=0$ (resp. si $R f=z f)$.

Pour $s_{0}$ fixé dans $S$ et $v_{m}$ à distance $m$ de $s_{o}$, on note $\left[s_{0}=v_{0}, v_{1}, v_{2}, \ldots, v_{m}\right]$ la géodésique joignant $s_{0}$ à $v_{m}$. Pour $m \geq 2$, on note $R^{*}\left(v_{m-1}\right)=\sum_{u} R\left(v_{m-1}, u\right)$ où la somme porte sur les $u$ tels que $d\left(v_{m-1}, u\right)=1, u \neq v_{m-2}$; on suppose $R^{*}\left(v_{m-1}\right)$ jamais nul (par exemple $R(s, t)>0$ si $d(s, t)=1)$. Pour $z \in \mathbb{C}^{*}$, on définit par récurrence la suite $\left(\eta_{k}\left(v_{m}\right)\right)_{k}$ (où $\eta_{k}\left(v_{m}\right)$ ne dépend que de $k, z$ et $v_{m-1}$ ) par:

$\eta_{m}\left(v_{m}\right)=1, \eta_{m-1}\left(v_{m}\right)=0, \eta_{m-2}\left(v_{m}\right)=\left(1-z^{-2}\right) R\left(v_{m-1}, v_{m-2}\right)\left[R^{*}\left(v_{m-1}\right)\right]^{-1}$ 
et

$\eta_{k}\left(v_{m}\right)=\left[-z^{-2} R\left(v_{m-1}, v_{m-2}\right) \eta_{k}\left(v_{m-2}\right)+\eta_{k}\left(v_{m-1}\right)\right]\left[R^{*}\left(v_{m-1}\right)\right]^{-1}, 0 \leq k \leq m-3$. On pose alors $H_{z} f\left(v_{m}\right)=z^{m} \sum_{k=0}^{k=m} \eta_{k}\left(v_{m}\right) f\left(v_{k}\right)$, pour $m \geq 0$. Par un simple
calcul on vérifie que:

$$
R * H_{z} f(s)=z^{d\left(s_{0}, s\right)+1} \Delta f(s)+z H_{z} f(s) ; \forall s \in S .
$$

En particulier $f$ est harmonique si et seulement si $H_{z} f$ est $z$-harmonique. L'opérateur $H_{z}$ est inversible, son inverse est donné par:

$$
H_{z}^{-1} g\left(v_{m}\right)=\sum_{k=0}^{k=m} \theta_{k}\left(v_{m}\right) z^{-k} g\left(v_{k}\right) ; \forall m \geq 0
$$

où $\theta_{m}\left(v_{m}\right)=1, \theta_{m-1}\left(v_{m}\right)=0, \theta_{m-2}\left(v_{m}\right)=-\eta_{m-2}\left(v_{m}\right)$ et pour $0 \leq k \leq m-3$, $\theta_{k}\left(v_{m}\right)=\left[\theta_{k}\left(v_{m-1}\right)-R\left(v_{m-1}, v_{m-2}\right) \theta_{k}\left(v_{m-2}\right)\right]\left[R^{*}\left(v_{m-1}\right)\right]^{-1}$.

Ce résultat concerne ce que l'on peut appeler le spectre formel de $R$, puisque l'on n'impose aucune condition de convergence à $f$ ou $H_{z} f$. Il n'a pas d'intérêt pour ce qui va nous préocuper maintenant c'est à dire l'étude du spectre $\ell^{2}$ de $R$. En effet lorsque $f$ est dans $\ell^{2}(S), H_{z} f$ n'est presque jamais dans $\ell^{2}(S)$.

Néanmoins pour $z=-1,\left(H_{-1} f\right)\left(v_{m}\right)=\left(H_{-1}^{-1} f\right)\left(v_{m}\right)=(-1)^{m} f\left(v_{m}\right)$; ainsi $H_{-1}$ est un opérateur $\ell^{2}$ (ou plus généralement $\ell^{r}$ ) involutif et on vérifie facilement qu'il transforme une fonction propre de $R$ pour la valeur propre $\gamma$ en une autre fonction propre pour la valeur propre $-\gamma$.

1.2. On considère dans ce paragraphe un groupe $\Lambda$ d'automorphismes de l'arbre transitif sur $S^{\prime}$ et un noyau $R$ à sauts de longueur 1 (plus précisément $R(s, t) \neq 0 \Leftrightarrow d(s, t)=1)$. On suppose de plus la condition suivante:

$$
\exists M \geq 0 / \forall u \in S ; \quad \sum_{s \in S}|R(s, u)| \leq M .
$$

On montre alors [K-R.2] (voir aussi [A]), que $R$ définit pour $r \geq 1$ un opérateur linéaire borné sur $\ell^{r}(S)$ et que pour $\gamma \in \mathbb{C},|\gamma|>\|R\|_{\text {cvr }}$ l'opérateur $R_{\gamma}=(\gamma-R)^{-1}$ est défini, plus précisément cet opérateur est donné par le noyau suivant:

$$
R_{\gamma}(s, s)=\frac{1}{2 w_{\gamma}(s)} \text { pour } s \in S
$$


$R_{\gamma}(s, t)=\frac{1}{2 w_{\gamma}(s)} \xi_{\gamma}\left(x_{n}=s, x_{n-1}\right) \cdots \xi_{\gamma}\left(x_{1}, x_{0}=t\right)$ si $d(s, t)=n \geq 1$ et si $[t, s]=\left[x_{0}=t, x_{1} \cdots, x_{n}=s\right]$ est la géodésique de $t$ à $s$;

où $w_{\gamma}(s)$ est une fonction complexe sur $S$ et $\xi_{\gamma}(s, t)$ une fonction complexe $\operatorname{sur}\{(s, t) \in S \times S / d(s, t)=1\}$.

Ces fonctions vérifient en outre les relations suivantes:

(1 bis) $\quad \forall t \in S, \gamma=2 w_{\gamma}(t)+\sum_{u / d(u, t)=1} \frac{w_{\gamma}(t)}{w_{\gamma}(u)} \xi_{\gamma}(u, t) R(t, u)$.

(2) $\quad \forall s, t \in S / d(s, t)=1 ; \quad \xi_{\gamma}(s, t)^{-1}-\xi_{\gamma}(t, s)=\frac{2 w_{\gamma}(t)}{R(s, t)}$.

(3) $\quad R_{\gamma}(s, t)=(-1)^{d(s, t)+1} R_{-\gamma}(s, t)$.

Autrement dit les fonctions $w_{\gamma}$ et $\xi_{\gamma}$ sont impaires en $\gamma$.

1.3. En supposant de plus la condition:

$\left(C_{r}\right)$ Le complémentaire du spectre $\ell^{r}$ de $R$ est connexe (pour un $r, 1 \leq r \leq 2$ ), les relations de 1.2 s'étendent par prolongement analytique sur C privé du spectre $\ell^{r}$ de $R$. Les fonctions $R_{\gamma}(s, t)$ sont holomorphes en $\gamma$; ainsi les fonctions $w_{\gamma}(s)=\frac{1}{2 R_{\gamma}(s, s)}$ et, pour $d(s, t)=1, \xi_{\gamma}(s, t)=$ $=2 w_{\gamma}(s) R_{\gamma}(s, t)$ sont méromorphes avec un pôle en $\gamma$ si et seulement si $R_{\gamma}(s, s)=0$.

Si 0 n'est pas dans le spectre $\ell^{r}$ de $R$, on a d'après $1.2(3) R_{0}(s, s)=0$; donc, pour tout $s \in S, w_{\gamma}(s)$ a un pôle en $\gamma=0$.

Proposition 1.4. Sous la condition $\left(C_{r}\right)$ de 1.3, soient $s, t \in S$ tels que $d(s, t)=1$ et que $w_{\gamma}(s)$ et $w_{\gamma}(t)$ aient un pôle en $\gamma_{0}$, n'appartenant pas au spectre $\ell^{r}$ de R. Alors:

1) ou $\xi_{\gamma}(s, t)$ et $\xi_{\gamma}(t, s)$ ont tous les deux un pôle en $\gamma=\gamma_{0}$ ou $\xi_{\gamma}(s, t)$ et $\xi_{\gamma}(t, s)$ tendent tous les deux vers 0 quand $\gamma$ tend vers $\gamma_{0}$.

2) Dans le cas d'un pôle on a l'équivalence suivante:

$$
\xi_{\gamma}(s, t) \sim \frac{-2 w_{\gamma}(s)}{R(t, s)} \text { et } \xi_{\gamma}(t, s) \sim \frac{-2 w_{\gamma}(t)}{R(s, t)} .
$$

3) Dans le cas d'un zéro, on a l'équivalence suivante:

$$
\xi_{\gamma}(s, t) \sim \frac{R(s, t)}{2 w_{\gamma}(t)} \text { et } \xi_{\gamma}(t, s) \sim \frac{R(t, s)}{2 w_{\gamma}(s)} .
$$


4) Si $u \in S$ vérifie $d(s, u)=2, d(t, u)=1$ et $w_{\gamma}(u)$ a un pôle en $\gamma_{0}$, alors $\xi_{\gamma}(s, t)$ et $\xi_{\gamma}(t, u)$ ne peuvent tous les deux avoir un pôle en $\gamma_{0}$.

5) Sous les hypothèses de 4$)$, si $\xi_{\gamma}(s, t)$ tend vers 0 et $\xi_{\gamma}(t, u)$ a un pôle alors $\xi_{\gamma}(s, t) \xi_{\gamma}(t, u)$ tend vers $\frac{-R(s, t)}{R(u, t)}$ quand $\gamma$ tend vers $\gamma_{0}$.

6) Sous les hypothèses de 4), si $\xi_{\gamma}(s, t)$ a un pôle et $\xi_{\gamma}(t, u)$ tend vers 0 , alors $\xi_{\gamma}(s, t) \xi_{\gamma}(t, u)$ est équivalent $\grave{a} \frac{-R(t, u)}{R(t, s)} \times \frac{w_{\gamma}(s)}{w_{\gamma}(u)}$ quand $\gamma$ tend vers $\gamma_{0}$.

7) Si $w_{\gamma}(v)$ a un pôle en $\gamma_{0}$, pour tout $v$, alors $\gamma_{0}=0$ et $\forall s \in S, \xi_{\gamma}(s, t)$ a un pôle pour un et un seul voisin $t$ de $s$.

DÉmonstration. D'après les formules (1), (1 bis) et (2) de 1.2, il existe $\varepsilon= \pm 1$ tel que:

$$
\begin{aligned}
& \xi_{\gamma}(s, t)=\frac{w_{\gamma}(s)}{R(t, s)}\left[-1+\varepsilon \sqrt{1+\frac{R(s, t) R(t, s)}{w_{\gamma}(s) w_{\gamma}(t)}}\right], \\
& \xi_{\gamma}(t, s)=\frac{w_{\gamma}(t)}{R(s, t)}\left[-1+\varepsilon \sqrt{1+\frac{R(s, t) R(t, s)}{w_{\gamma}(s) w_{\gamma}(t)}}\right] .
\end{aligned}
$$

Le comportement de ces fonctions en $\gamma=\gamma_{0}$ ne dépend que de $\varepsilon$, et on en déduit facilement les résultats 1), 2) et 3 ).

4) Supposons qu'il y ait deux pôles, alors:

$$
R_{\gamma}(s, u)=\frac{1}{2 w_{\gamma}(s)} \xi_{\gamma}(s, t) \xi_{\gamma}(t, u) \sim \frac{-\xi_{\gamma}(t, u)}{R(t, s)}
$$

c'est contradictoire avec le fait que $R_{\gamma}(s, u)$ est bornée.

5 ) et 6) se prouvent simplement à partir de 2) et 3).

7) D'après 1.2.1, $\gamma=2 w_{\gamma}(s)+\sum_{t \in \Sigma(s, 1)} \xi_{\gamma}(s, t) R(t, s)$.

Comme $w_{\gamma}(s)$ a un pôle, la fonction $\xi_{\gamma}(s, t)$ a un pôle pour au moins un $t \in \Sigma(s, 1)$ et en fait un seul d'après 4$)$, notons le $t_{0}$.

D'après 1.2.2, $2 w_{\gamma}(s)=R\left(t_{0}, s\right)\left[\xi_{\gamma}\left(t_{0}, s\right)^{-1}-\xi_{\gamma}\left(s, t_{0}\right)\right]$. Donc:

$$
\begin{aligned}
\gamma & =R\left(t_{0}, s\right)\left[\xi_{\gamma}\left(t_{0}, s\right)^{-1}-\xi_{\gamma}\left(s, t_{0}\right)\right]+\sum_{t} \xi_{\gamma}(s, t) R(t, s) \\
& =R\left(t_{0}, s\right) \xi_{\gamma}\left(t_{0}, s\right)^{-1}+\sum_{t \neq t_{0}} \xi_{\gamma}(s, t) R(t, s)
\end{aligned}
$$

et le membre de droite tend vers 0 quand $\gamma$ tend vers $\gamma_{0}$, donc $\gamma_{0}=0$. 
Remarque 1.5. 1) Si $R^{2}$ est symétrique réel ( $\operatorname{sur} S^{\prime}$ ou $S^{\prime \prime}$ ) alors le spectre de $R^{2}$ agissant sur $\ell^{2}\left(S^{\prime}\right)$ ou $\ell^{2}\left(S^{\prime \prime}\right)$ est réel (borné). D'après [K-R.2; 7.2] le spectre $\ell^{2}$ de $R$ est borné et contenu dans la réunion des axes réel et imaginaire. Ainsi la condition $\left(C_{2}\right)$ de 1.3 est vérifiée.

2) Supposons que $R$ est à valeurs réelles ou imaginaires pures et que le groupe $\Lambda$ vérifie la condition suivante, (cf. [K-R.2; 5]):

(M) $\forall s, t \in S^{\prime} / d(s, t)=2$, il existe $\lambda \in \Lambda$ fixant le milieu de $[s, t]$ tel que $\lambda(s)=t$,

alors le noyau $R^{2}$ est réel symétrique sur $S^{\prime}$ car si $s \in S^{\prime \prime}$ et $t, t^{\prime} \in \Sigma(s, 1)$ on a $R(t, s)=R\left(t^{\prime}, s\right)$ et $R(s, t)=R\left(s, t^{\prime}\right)$ donc $R^{2}\left(t, t^{\prime}\right)=R^{2}\left(t^{\prime}, t\right)$.

De plus 0 est dans le spectre $\ell^{2}$ de $R$ (et même le spectre $\ell^{r}$ si la condition $\left(C_{r}\right)$ est vérifiée): sinon, on a vu en 1.3 que $w_{\gamma}(s)$ a un pôle en $\gamma=0, \forall s \in S$; d'après 1.4.7, pour $s \in S^{\prime \prime}$, il existe $t \in \Sigma(s, 1)$ tel que $\xi_{\gamma}(s, t)$ a un pôle en $\gamma=0$; il en est de même de $\xi_{\gamma}\left(s, t^{\prime}\right) \quad \forall t^{\prime} \in \Sigma(s, 1)$ d'après $(M)$ et aussi de $\xi_{\gamma}\left(t^{\prime}, s\right)$ d'après 1.4.1; ce qui contredit le résultat 1.4.4.

\subsection{Calcul du noyau de Green en 0: (0 supposé hors du spectre $\left.\ell^{r}\right)$.}

D'après ce qui précéde tout sommet $s \in S$ a un unique sommet voisin $s^{\prime}$ tel que $\xi_{\gamma}\left(s, s^{\prime}\right)$ ait un pôle en 0 , alors $\xi_{\gamma}\left(s^{\prime}, s\right)$ a aussi un pôle et donc $\left(s^{\prime}\right)^{\prime}=s$. On dit que l'arête $\left\{s, s^{\prime}\right\}$ est spéciale.

On choisit $s_{0} \in S^{\prime}$, on note $s_{1}=s_{0}^{\prime}$ et $s_{2}, \cdots, s_{q+1}$ les autres voisins de $s_{0}$. Les voisins de $s_{i}$ sont $s_{i}^{j}, \quad 1 \leq j \leq \ell_{i}+1$. On suppose encore $s_{i}^{1}=\left(s_{i}\right)^{\prime}$, donc $s_{1}^{1}=s_{0}$ et $s_{i}^{1} \neq s_{0}$ pour $2 \leq i \leq q+1$.

Si $s, t \in S$ avec $d(s, t)=n$, on note $[t, s]=\left(x_{0}=t, \cdots, x_{n}=s\right)$ et alors :

$$
R_{\gamma}(s, t)=\frac{1}{2 w_{\gamma}(s)} \xi_{\gamma}\left(x_{n}, x_{n-1}\right) \cdots \xi_{\gamma}\left(x_{1}, x_{0}\right) .
$$

D'après 1.4.4 dans le produit des $\xi_{\gamma}\left(x_{i}, x_{i-1}\right)$ deux pôles ne peuvent se suivre. On peut donc considérer que $R_{\gamma}$ est le produit des quantités suivantes:

1) ou $\frac{1}{2 w_{\gamma}(s)}$ qui tend vers zéro ou $\frac{1}{2 w_{\gamma}(s)} \xi_{\gamma}\left(s, x_{n-1}\right)$ qui tend vers une constante car $\xi_{\gamma}\left(s, x_{n-1}\right)$ a un pôle;

2) puis des facteurs de la forme:

ou $\xi_{\gamma}\left(x_{i}, x_{i-1}\right)$ qui tend vers zéro ou $\xi_{\gamma}\left(x_{i}, x_{i-1}\right) \xi_{\gamma}\left(x_{i-1}, x_{i-2}\right)$ qui tend vers une constante non nulle car $\xi_{\gamma}\left(x_{i}, x_{i-1}\right)$ tend vers zéro et $\xi_{\gamma}\left(x_{i-1}, x_{i-2}\right)$ a un pôle. 
Ainsi la limite de $R_{\gamma}(s, t)$ est nulle sauf si $n=2 p+1, \xi_{\gamma}\left(x_{2 p+1}, x_{2 p}\right)$ a un pôle, $\xi_{\gamma}\left(x_{2 j}, x_{2 j-1}\right)$ tend vers zéro et $\xi_{\gamma}\left(x_{2 j-1}, x_{2 j-2}\right)$ a un pôle $\forall j, 1 \leq j \leq p$. Autrement dit les arêtes $\left(x_{2 j-1}, x_{2 j-2}\right)$ sont spéciales $\forall j, 1 \leq j \leq p+1$ et les autres non.

Dans ce cas d'après 1.4.5, on obtient à la limite:

$$
R_{0}(s, t)=\frac{(-1)^{p+1}}{R\left(x_{2 p}, s\right)} \prod_{k=1}^{p} \frac{R\left(x_{2 k}, x_{2 k-1}\right)}{R\left(x_{2 k-2}, x_{2 k-1}\right)} .
$$

Proposition 1.7. On suppose vérifiée la condition $\left(C_{r}\right)$ et que 0 n'est pas dans le spectre $\ell^{r}(1 \leq r<\infty)$ de $R$, alors:

1) Le groupe $\Lambda$ laisse invariant l'ensemble d'arêtes spéciales, en particulier X est semi-homogène et $\Lambda$ est transitif sur $S^{\prime}$ et $S^{\prime \prime}$.

2) Fixons une arête spéciale $\left\{s^{\prime}, s^{\prime \prime}\right\}$ avec $s^{\prime}$ dans $S^{\prime}$ et on note:

$\Sigma\left(s^{\prime}, 1\right)=\left\{s_{1}^{\prime \prime}=s^{\prime \prime}, \cdots, s_{q+1}^{\prime \prime}\right\}, \quad \Sigma\left(s^{\prime \prime}, 1\right)=\left\{s_{1}^{\prime}=s^{\prime}, \cdots, s_{\ell+1}^{\prime}\right\}$ et pour $s, t \in S / d(s, t)=1$ :

$$
\begin{aligned}
& R(s, t)=p_{i}^{\prime} \text { si } t \in S^{\prime}, s \in S^{\prime \prime} \text { et }\{s, t\} \quad \Lambda \text {-conjuguée à }\left\{s_{i}^{\prime \prime}, s^{\prime}\right\} \\
& R(s, t)=p_{i}^{\prime \prime} \text { si } t \in S^{\prime \prime}, s \in S^{\prime} \text { et }\{s, t\} \quad \Lambda \text {-conjuguée à }\left\{s_{i}^{\prime}, s^{\prime \prime}\right\} .
\end{aligned}
$$

On a alors:

$$
\left|p_{1}^{\prime \prime}\right|^{r}>\sum_{i=2}^{\ell+1}\left|p_{i}^{\prime \prime}\right|^{r} \text { et }\left|p_{1}^{\prime}\right|^{r}>\sum_{i=2}^{q+1}\left|p_{i}^{\prime}\right|^{r}
$$

DÉmonstration. 1) D'après 1.6 des arêtes spéciales sont bien définies intrinséquement par $R$, leur ensemble est donc stable par $\Lambda$ et la transitivité de $\Lambda$ sur $S^{\prime \prime}$ découle de celle sur $S^{\prime}$; en particulier l'arbre est semihomogène.

2) Cela résulte des calculs de [K-R.1; 3.5].

Remarque 1.8. Si $\Lambda$ vérifie la condition 1.7.1 et la condition $(U)$ de [B.K] ou [K-R.1] suivante:

( $U$ ) Il existe un sous-ensemble $\Lambda_{2}$ de $\Lambda$, tel que l'application $\lambda \longmapsto \lambda s_{0}$ induise des bijections de $\Lambda_{2}$ sur $\Sigma\left(s_{0}, 2\right)$ et de $\Lambda_{2}^{-1} \operatorname{sur} \Sigma\left(s_{0}, 2\right)$,

on peut montrer que l'arbre est nécessairement homogène; alors on a vu en [K-R.1; 3.6] que le noyau $R_{0}$ donné par l'expression de 1.6 définit un opérateur $\ell^{r}$ si et seulement si les deux conditions finales de 1.7.2 sont vérifiées. Sous ces hypothèses sur $\Lambda$, il y a donc équivalence entre ces deux conditions de 1.7.2 et la non appartenance de 0 au spectre $\ell^{r}$ de $R$. 


\section{Etude du spectre pour certains noyaux sur $S^{\prime}$.}

2.1. On considère dans tout ce paragraphe un noyau réel $R^{\prime}$ à sauts de longueur 0 ou 2 sur la partie $S^{\prime}$ de l'ensemble des sommets d'un arbre localement fini, on impose en fait que:

$$
\forall s, t \in S^{\prime}, d(s, t)=2 \Rightarrow R^{\prime}(s, t) \neq 0 .
$$

On suppose de plus $R^{\prime}$ invariant par un groupe $\Lambda$ transitif sur $S^{\prime}$ et satisfaisant à la condition suivante, (cf. [K.R.2; 6]):

$\left(M^{\prime}\right) \forall s \in S^{\prime \prime}$, le fixateur $\Lambda_{s}$ de $s$ dans $\Lambda$ est doublement transitif sur $\Sigma(s, 1)$.

D'après [K-R.2; 6] le noyau $R^{\prime}$ est symétrique, son spectre $\ell^{2}$ est donc réel borné. En particulier le complémentaire de ce spectre est connexe.

Les résultats qui suivent sont encore valables pour le spectre $\ell^{r},(1 \leq r<\infty)$ sous la condition suivante:

$\left(C_{r}^{\prime}\right)$ Le complémentaire du spectre $\ell^{r}$ de $R^{\prime}$ est connexe.

2.2. Sous ces conditions et pour $\gamma \in \mathbb{C},|\gamma|>\left\|R^{\prime}\right\|_{c v r},(r \geq 1)$, on a donné en $[\mathrm{K}-\mathrm{R} .2 ; 6]$ l'expression de l'opérateur $\left(\gamma-R^{\prime}\right)^{-1}=R_{\gamma}^{\prime}$ :

$R_{\gamma}^{\prime}(s, s)=\frac{1}{2 \alpha_{\gamma}(s)}$ et $R_{\gamma}^{\prime}(s, t)=\frac{1}{2 \alpha_{\gamma}(s)} \eta_{\gamma}\left(x_{n}=s, x_{n-1}\right) \cdots \eta_{\gamma}\left(x_{1}, x_{0}=t\right)$, si $d(s, t)=2 n, n \geq 1$ et si la géodésique joignant $t$ à $s$ formée d'élements de $S^{\prime}$ s'écrit $[t, s]^{\prime}=\left([t, s] \cap S^{\prime}\right)=\left(x_{0}=t, x_{1} \cdots, x_{n}=s\right)$; où $\alpha_{\gamma}(s)$ est une fonction complexe sur $S^{\prime}$ et $\eta_{\gamma}(s, t)$ une fonction complexe sur $\left\{(s, t) \in S^{\prime} \times S^{\prime} / d(s, t)=2\right\}$.

Ces expressions se prolongent analytiquement en $\gamma$ sur $\mathbb{C}$ privé du spectre $\ell^{r}$ de $R^{\prime}$. Les fonctions $R_{\gamma}^{\prime}(s, t)$ sont holomorphes en $\gamma$; ainsi les fonctions $\alpha_{\gamma}(s)=\frac{1}{2 R_{\gamma}^{\prime}(s, s)}$ et pour $d(s, t)=2, \eta_{\gamma}(s, t)=2 \alpha_{\gamma}(s) R_{\gamma}^{\prime}(s, t)$ sont méromorphes avec un pôle en $\gamma$ seulement si $R_{\gamma}^{\prime}(s, s)=0$.

On rappelle qu'en [K-R.2] on a montré que les fonctions $\alpha_{\gamma}(s)$ ne dépendent pas de $s$ et que $\eta_{\gamma}(s, t)=\eta_{\gamma}(t, s)$ ne dépendent que de $u \in[s, t] \cap S^{\prime \prime}$. On a choisi $s_{0} \in S^{\prime}$ et noté $\Sigma\left(s_{0}, 1\right)=\left\{s_{1}, \cdots, s_{q+1}\right\}$ et $\Sigma\left(s_{0}, 2\right)=$ $=\left\{s_{i, j} / 1 \leq i \leq q+1,1 \leq j \leq \ell_{i}\right\}$ avec $d\left(s_{i}, s_{i, j}\right)=1$ et $d\left(s_{0}, s_{i, j}\right)=2$. On pose alors:

$$
\begin{aligned}
& \alpha(\gamma)=\alpha_{\gamma}(s) \forall s \in S^{\prime} \text { et } \eta_{i}(\gamma)=\eta_{\gamma}\left(s_{i, j}, s_{0}\right)=\eta_{\gamma}\left(s_{0}, s_{i, j}\right) \text {, de plus } \beta_{0}^{\prime}=R^{\prime}\left(s_{0}, s_{0}\right) \\
& \text { et } \beta_{i}^{\prime}=R^{\prime}\left(s_{0}, s_{i, j}\right)=R^{\prime}\left(s_{i, j}, s_{0}\right) .
\end{aligned}
$$


On a alors obtenu les relations suivantes:

(1) $\gamma=2 \alpha(\gamma)+\beta_{0}^{\prime}+\sum_{i=1}^{q+1} \ell_{i} \eta_{i}(\gamma) \beta_{i}^{\prime}$.

(2) $\eta_{i}^{-1}(\gamma)-\eta_{i}(\gamma)=\frac{2 \alpha(\gamma)}{\beta_{i}^{\prime}}+\left(\ell_{i}-1\right)\left(\eta_{i}(\gamma)-1\right)$.

(3) $\eta_{i}(\gamma)=\frac{-2 \alpha(\gamma)+\left(\ell_{i}-1\right) \beta_{i}^{\prime} \pm \sqrt{\left[2 \alpha(\gamma)-\left(\ell_{i}-1\right) \beta_{i}^{\prime}\right]^{2}+4 \ell_{i}{\beta_{i}^{\prime}}^{2}}}{2 \ell_{i} \beta_{i}^{\prime}}$.

Proposition 2.3. Soit $\gamma_{0} \in \mathbb{C}$ non dans le spectre $\ell^{r}$ de $R$. On suppose que $R_{\gamma_{0}}^{\prime}\left(s_{0}, s_{0}\right)=0$ i.e. $\alpha(\gamma)$ a un pôle en $\gamma_{0}$. Alors

1) ou $\eta_{i}(\gamma)$ a un pôle en $\gamma_{0}$ et alors $\eta_{i}(\gamma) \sim \frac{-2 \alpha(\gamma)}{\ell_{i} \beta_{i}^{\prime}}$. ou $\eta_{i}(\gamma)$ tend vers 0 quand $\gamma$ tend vers $\gamma_{0}$ et alors $\eta_{i}(\gamma) \sim \frac{\beta_{i}^{\prime}}{2 \alpha(\gamma)}$.

2) Pour $i \neq j, \eta_{i}(\gamma)$ et $\eta_{j}(\gamma)$ ne peuvent simutanément avoir des pôles et $\eta_{i}(\gamma) \eta_{j}(\gamma)$ tend vers 0 ou une constante.

3) Il existe $i_{0}$ (unique) tel que $\eta_{i_{0}}(\gamma)$ ait un pôle.

4) $\gamma_{0}=\beta_{0}^{\prime}+\left(\ell_{i_{0}}-1\right) \beta_{i_{0}}^{\prime}$.

N.B: Dans la suite on numérote de façon que $i_{0}=1$.

DÉmonstration. 1) D’après 2.2 (3) on a:

$$
\eta_{i}(\gamma)=\frac{-2 \alpha(\gamma)+\left(\ell_{i}-1\right) \beta_{i}^{\prime}}{2 \ell_{i} \beta_{i}^{\prime}}\left[1 \pm \sqrt{1+4 \ell_{i}\left(\frac{\beta_{i}^{\prime}}{2 \alpha(\gamma)-\left(\ell_{i}-1\right) \beta_{i}^{\prime}}\right)^{2}}\right] .
$$

Selon le signe on obtient bien les résultats annoncés.

2) $R_{\gamma}^{\prime}\left(s_{i, k}, s_{j, m}\right)=\frac{1}{2 \alpha(\gamma)} \eta_{\gamma}\left(s_{i, k}, s_{0}\right) \eta_{\gamma}\left(s_{0}, s_{j, m}\right)=\frac{\eta_{i}(\gamma) \eta_{j}(\gamma)}{2 \alpha(\gamma)}$. Si $\eta_{i}(\gamma)$ et $\eta_{j}(\gamma)$ ont un pôle, $R_{\gamma}^{\prime}\left(s_{i, k}, s_{j, m}\right) \sim \frac{2 \alpha(\gamma)}{\ell_{i} \ell_{j} \beta_{i}^{\prime} \beta_{j}^{\prime}}$. Ceci contredit le fait que $R_{\gamma}^{\prime}$ est
bornée.

3) On a d'après $2.2(1) \gamma=2 \alpha(\gamma)+\beta_{0}^{\prime}+\sum_{i} \ell_{i} \eta_{i}(\gamma) \beta_{i}^{\prime}$. Comme $\alpha(\gamma)$ a un pôle en $\gamma_{0}$, il en est de même de l'un des $\eta_{i}(\gamma)$. L'unicité découle de 2$)$.

4) De $2.2(2)$ on tire $2 \alpha(\gamma)=\eta_{i}^{-1}(\gamma) \beta_{i}^{\prime}-\ell_{i} \eta_{i}(\gamma) \beta_{i}^{\prime}+\left(\ell_{i}-1\right) \beta_{i}^{\prime}$ donc $\gamma=\beta_{0}^{\prime}+\left(\ell_{i_{0}}-1\right) \beta_{i_{0}}^{\prime}+\eta_{i_{0}}^{-1}(\gamma) \beta_{i_{0}}^{\prime}+\sum_{i \neq i_{0}} \ell_{i} \eta_{i}(\gamma) \beta_{i}^{\prime}$. Les derniers termes tendent vers 0 quand $\gamma$ tend vers $\gamma_{0}$, donc $\gamma_{0}=\beta_{0}^{\prime}+\left(\ell_{i_{0}}-1\right) \beta_{i_{0}}^{\prime}$.

\subsection{Calcul du noyau de Green.}

On se place en $\gamma_{0}$ non dans le spectre $\ell^{r}$ de $R^{\prime}$ et tel que $R_{\gamma_{0}}^{\prime}\left(s_{0}, s_{0}\right)=0$. Le noyau de Green est déterminé par la fonction de Green $g_{\gamma_{0}}^{\prime}(s)=R_{\gamma_{0}}^{\prime}\left(s, s_{0}\right)$. 
On vient de voir qu'il existe une classe de sommets "spéciaux" dans $S^{\prime \prime}$, chaque sommet $s_{0} \in S^{\prime}$ a un et un seul voisin spécial noté $s_{1}$.

On calcule facilement $g_{\gamma_{0}}^{\prime}$ à partir de 2.3 (cf. 1.6):

- $g_{\gamma_{0}}^{\prime}(s)$ est nul si $s \in S^{\prime}$ et $d\left(s, s_{0}\right)$ est multiple de 4 .

- Si $d\left(s, s_{0}\right)=4 n+2$, on note $\left(x_{0}=s_{0}, x_{1}, \cdots, x_{4 n+2}=s\right)$ la géodésique de $s_{0}$ à $s$, alors $g_{\gamma_{0}}^{\prime}(s)$ est nul sauf si les sommets $\left(x_{4 k+1}\right)_{0 \leq k \leq n}$ sont spéciaux; dans ce cas pour $0 \leq k \leq n-1$, on choisit $\lambda_{k} \in \Lambda$ tel que $\lambda_{k}\left(x_{4 k+2}\right)=s_{0}$ et alors $\lambda_{k}\left(x_{4 k+3}\right)=s_{j_{k}}$ avec $2 \leq j_{k} \leq q+1$ et l'on a:

$$
g_{\gamma_{0}}^{\prime}(s)=\frac{-1}{\ell_{1} \beta_{1}^{\prime}} \prod_{k=0}^{n-1}\left(\frac{-\beta_{j_{k}}^{\prime}}{\ell_{1} \beta_{1}^{\prime}}\right) .
$$

LEMME 2.5. On suppose qu'il existe une classe de sommets spéciaux dans $S^{\prime \prime}$, stable par $\Lambda$ et telle que tout sommet $s_{0} \in S^{\prime}$ a un et un seul voisin spécial $s_{1}$. On définit une fonction $g_{\gamma_{0}}^{\prime}$ sur $S^{\prime}$ par les formules ci-dessus et alors:

$$
\sum_{s \in \Sigma\left(s_{0}, 4 n+2\right)}\left|g_{\gamma_{0}}^{\prime}(s)\right|^{r}=\frac{\ell_{1}}{\left|\ell_{1} \beta_{1}^{\prime}\right|^{r}}\left[\sum_{j \geq 2} \ell_{1} \ell_{j}\left|\frac{\beta_{j}^{\prime}}{\mid \ell_{1} \beta_{1}^{\prime}}\right|^{r}\right]^{n} .
$$

En particulier $g_{\gamma_{0}}^{\prime} \in \ell^{r}\left(S^{\prime}\right) \Longleftrightarrow \ell_{1}^{r-1}\left|\beta_{1}^{\prime}\right|^{r}>\sum_{j \geq 2} \ell_{j}\left|\beta_{j}^{\prime}\right|^{r}$.

DÉmonstration. C'est la même démonstration que celle de [K-R.1;3.5] en tenant compte de ce que pour $s \in S^{\prime}$ il y a $\ell_{1}$ (resp. $\ell_{j}$ ) sommets $t \in \Sigma(s, 2)$ tels que le milieu de $[s, t]$ soit spécial (resp. non spécial «de type $j »)$.

THÉorème 2.6. On suppose $1 \leq r \leq 2$. Soit $\gamma_{0} \in \mathrm{C}$. $s_{0}$ tel que

1) $S i \gamma_{0} \notin s p_{r}\left(R^{\prime}\right)$ et $R_{\gamma_{0}}^{\prime}\left(s_{0}, s_{0}\right)=0$; il existe un (unique) voisin $s_{1} d e$

$$
\gamma_{0}=\beta_{0}^{\prime}+\left(\ell_{1}-1\right) \beta_{1}^{\prime} \text { et } \ell_{1}^{r-1}\left|\beta_{1}^{\prime}\right|^{r}>\sum_{j \geq 2} \ell_{j}\left|\beta_{j}^{\prime}\right|^{r}
$$

et le noyau $R_{\gamma_{0}}^{\prime}\left(s, s_{0}\right)$ est donné par les formules de 2.4 .

2) Réciproquement, si $\ell_{1}^{r-1}\left|\beta_{1}^{\prime}\right|^{r}>\sum_{j \geq 2} \ell_{j}\left|\beta_{j}^{\prime}\right|^{r}$ et si le groupe $\Lambda$ d'invariance vérifie la condition $(U)$ de 1.8 , alors $\gamma_{0}=\beta_{0}^{\prime}+\left(\ell_{1}-1\right) \beta_{1}^{\prime} \notin s p_{r}\left(R^{\prime}\right)$ et $R_{\gamma_{0}}^{\prime}\left(s_{0}, s_{0}\right)=0$.

Remarque. Pour $\ell_{i}=1, \forall i$ et $\beta_{0}^{\prime}=0$, on retrouve une partie des résultats du $\S 1$ (pour l'arbre formé des sommets de $S^{\prime}$ ); la condition d'invariance du noyau est alors exactement celle de [Ft-S]. 
DÉmonstration. Cela résulte des résultats précédents et de raisonnements exactement analogues à ceux de 1.7 et 1.8. Il faut juste noter que, comme $1 \leq r \leq 2, \ell_{i}\left|\beta_{i}^{\prime}\right|^{r} \geq \ell_{i}^{r-1}\left|\beta_{i}^{\prime}\right|^{r}$ et donc l'inégalité $\ell_{i}^{r-1}\left|\beta_{i}^{\prime}\right|^{r}>\sum_{j \neq i} \ell_{j}\left|\beta_{j}^{\prime}\right|^{r}$ ne peut avoir lieu que pour un seul $i(1 \leq i \leq q+1)$ que l'on note $i=1$.

2.7. On se propose maintenant de déterminer des conditions sous lesquelles un complexe $\gamma$ n'est pas dans $s p_{r}\left(R^{\prime}\right)$; s'il existe $i$ tel que $\ell_{i}^{r-1}\left|\beta_{i}^{\prime}\right|^{r}>\sum_{j \neq i} \ell_{j}\left|\beta_{j}^{\prime}\right|^{r}$ on suppose $\gamma \neq \gamma_{0}=\beta_{0}^{\prime}+\left(\ell_{i}-1\right) \beta_{i}^{\prime}$.

D'après ce qui précéde pour $\gamma \notin s p_{r}\left(R^{\prime}\right)$ et $\gamma \neq \gamma_{0}$, il existe des nombres complexes $\alpha \neq 0,\left(\eta_{i}(\gamma)\right)_{1 \leq i \leq q+1}$ tels que $R_{\gamma}^{\prime}$ est le noyau $H^{\prime}(s, t)$ invariant par $\Lambda$, tel que

$$
H^{\prime}(s, t)=\frac{1}{2 \alpha} \eta_{i_{n}} \cdots \eta_{i_{1}} \text { si } d(s, t)=2 n \text { et pour }[s, t]^{\prime}=\left(x_{n}=s, \cdots, x_{0}=t\right)
$$

quand $\lambda_{j} \in \Lambda$ vérifie $\lambda_{j}\left(x_{j-1}\right)=s_{0}$ alors le milieu de $\left[\lambda_{j}\left(x_{j-1}\right), \lambda_{j}\left(x_{j}\right)\right]$ est $s_{i_{j}}\left(1 \leq i_{j} \leq q+1\right)$.

On peut noter que forcément les $\eta_{i}$ sont les mêmes pour les $i$ correspondant à une orbite du fixateur $\Lambda_{0}$ de $s_{0}$ dans $\left\{s_{1}, \cdots, s_{q+1}\right\}$.

Lemme 2.8. Pour un noyau $H$ sur $S^{\prime} \times S^{\prime}$, invariant par $\Lambda$, on définit la norme droite de classe $r,\|H\|_{d r}$ de $H$ par:

$$
\|H\|_{d r}^{r}=\sum_{s \in S^{\prime}}\left|H\left(s, s_{0}\right)\right|^{r} .
$$

On peut calculer la norme droite du noyau $H^{\prime}$ abstraitement défini par les formules ci-dessus:

$$
\begin{aligned}
\left\|H^{\prime}\right\|_{d r}^{r} & =\left|\frac{1}{2 \alpha}\right|^{r}\left(1-\sum_{i=1}^{q+1} \frac{\ell_{i}\left|\eta_{i}\right|^{r}}{1+\ell_{i}\left|\eta_{i}\right|^{r}}\right)^{-1} \text { si cette expression est positive, } \\
\left\|H^{\prime}\right\|_{d r}^{r} & =\infty \text { sinon. }
\end{aligned}
$$

DÉMONSTRATION. On voit facilement sur la définition ci-dessus de $H^{\prime}$ que:

$$
\left\|H^{\prime}\right\|_{d r}^{r}=\left|\frac{1}{2 \alpha}\right|^{r} \sum_{n=0}^{\infty}\left[\sum \ell_{i_{1}} \cdots \ell_{i_{n}}\left|\eta_{i_{1}}\right|^{r} \cdots\left|\eta_{i_{n}}\right|^{r}\right]=\frac{A}{|2 \alpha|^{r}}
$$

où la seconde somme porte sur les $\left(i_{1}, \cdots, i_{n}\right) \in\{1, \cdots, q+1\}^{n}$ tels que $i_{m} \neq i_{m+1}$ pour $1 \leq m<n$.

On refait le même raisonnement que T. Steger dans [St] ou [Ft.S] sur l'expression abstraite de $A$, on montre que $A$ est fini si et seulement si $\sum_{i=1}^{q+1} \frac{\ell_{i}\left|\eta_{i}\right|^{r}}{1+\ell_{i}\left|\eta_{i}\right|^{r}}<1$ et que $A=\left(1-\sum_{i=1}^{q+1} \frac{\ell_{i}\left|\eta_{i}\right|^{r}}{1+\ell_{i}\left|\eta_{i}\right|^{r}}\right)^{-1}$. 
LEMME 2.9. On suppose que $1 \leq r \leq 2$ et que le groupe 1 d'invariance satisfait à la condition $(U)$ de 1.8 alors le noyau $H^{\prime}$ de 2.7 est un opérateur borné sur $\ell^{r}\left(S^{\prime}\right)$ si et seulement si $\sum_{i=1}^{q+1} \frac{\ell_{i}\left|\eta_{i}\right|^{r}}{1+\ell_{i}\left|\eta_{i}\right|^{r}}<1$.

DÉmonstration. Si $H^{\prime}$ est un opérateur borné $\ell^{r}, H^{\prime} * \delta_{s_{0}} \in \ell^{r}\left(S^{\prime}\right)$ or $\left\|H^{\prime} * \delta_{s_{0}}\right\|_{r}^{r}=\left\|H^{\prime}\right\|_{d r}^{r}$ par définition donc d'après le lemme 2.8 on a bien $\sum_{i=1}^{q+1} \frac{\ell_{i}\left|\eta_{i}\right|^{r}}{1+\ell_{i}\left|\eta_{i}\right|^{r}}<1$.

Réciproquement supposons que cette condition est vérifiée. Pour $z \in \mathrm{C} /|z|<1$, on définit (avec les notations de 2.8):

$f_{z}(s)=z^{d\left(s, s_{0}\right)}\left|H^{\prime}\left(s, s_{0}\right)\right|^{r}$ et $F(z)=\sum_{s \in S^{\prime}} f_{z}(s)=\left|\frac{1}{2 \alpha}\right|^{r}\left(1-\sum_{i=1}^{q+1} \frac{z^{2} \ell_{i}\left|\eta_{i}\right|^{r}}{1+z^{2} \ell_{i}\left|\eta_{i}\right|^{r}}\right)^{-1}$.

Mais par hypothèse $F(z)$ n'a pas de pôle dans $\{z \in \mathbb{C} /|z| \leq 1\}$, le rayon de convergence de la série définissant $F(z)$ est strictement plus grand que 1.

Ainsi $\sum_{s \in \Sigma\left(s_{0}, 2 n\right)}\left|H^{\prime}\left(s, s_{0}\right)\right|^{r}$ décroit en exponentielle en $n$. L'hypothèse $d u$ théorème 2.3 [K-R.1] est donc vérifiée et $H^{\prime}$ est un opérateur borné sur $\ell^{r}\left(S^{\prime}\right)$.

LEMME 2.10. Le noyau $H^{\prime}$ abstraitement défini en 2.7 est un inverse formel de $\gamma-R^{\prime}$ si et seulement si:

$$
\begin{aligned}
& \gamma=2 \alpha+\beta_{0}^{\prime}+\sum_{i=1}^{q+1} \ell_{i} \eta_{i} \beta_{i}^{\prime} \text { et } \\
& \frac{2 \alpha}{\beta_{i}^{\prime}}=-\ell_{i} \eta_{i}+\eta_{i}^{-1}+\left(\ell_{i}-1\right), \text { pour tout } i,(1 \leq i \leq q+1) .
\end{aligned}
$$

DÉmonstration. Cela résulte des équations obtenues dans [K-R.2;6], car dans le cas présent (1) et (1)bis équivalent à la première relation et (2) et ( 2 bis) à la seconde.

Remarque 2.11. Supposons que pour $1 \leq i \neq j \leq q+1, \ell_{i}=\ell_{j}$ et $\beta_{i}^{\prime}=\beta_{j}^{\prime}$, alors si $\eta_{i} \neq \eta_{j}, \quad \eta_{i} \eta_{j}$ est entièrement déterminé par la seconde équation de 2.10: on a $\eta_{i} \eta_{j}=-\frac{1}{\ell_{i}}$ donc $\ell_{i}\left|\eta_{i}\right|^{r} \ell_{j}\left|\eta_{j}\right|^{r}=\ell_{i}^{2-r}$ qui est supérieur à 1 si $r \leq 2$. Le début des calculs de 2.8 montre alors que $\left\|H^{\prime}\right\|_{d r}^{r}=\infty$. Ainsi d'après $2.8,2.9$ et 2.10 si $H^{\prime}$ est un opérateur borné inverse de $\gamma-R^{\prime}$ et si, pour $i \neq j,\left(\ell_{i}, \beta_{i}^{\prime}\right)=\left(\ell_{j}, \beta_{j}^{\prime}\right)$ alors $\eta_{i}=\eta_{j}$. 
Proposition 2.12. On suppose que $\Lambda$ vérifie la condition $(U)$ de 1.8 et que $1 \leq r \leq 2$. Alors le complémentaire du spectre $\ell^{r}$ de $R^{\prime}$ éventuellement

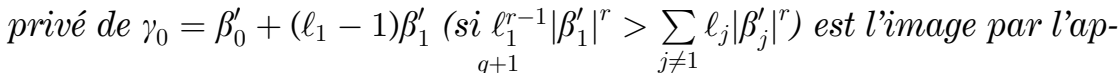
plication $\gamma\left(\alpha,\left(\eta_{i}\right)\right)=2 \alpha+\beta_{0}^{\prime}+\sum_{i=1}^{q+1} \ell_{i} \eta_{i} \beta_{i}^{\prime}$ de l'ensemble des $\left(\alpha,(\eta)_{i}\right) \in \mathbb{C}^{q+2}$
vérifiant:
a) $-\ell_{i} \eta_{i}+\eta_{i}^{-1}+\left(\ell_{i}-1\right)=\frac{2 \alpha}{\beta_{i}^{\prime}}$, pour tout $i, 1 \leq i \leq q+1$.
b) $\sum_{i=1}^{q+1} \frac{\ell_{i}\left|\eta_{i}\right|^{r}}{1+\ell_{i}\left|\eta_{i}\right|^{r}}<1$.

DÉmonstration. Cela résulte aussitôt de 2.7, 2.9 et 2.10 .

Corollaire 2.13. Sous les conditions de 2.12 et pour $r=2$, un $\gamma_{1} \in \mathbb{R}\left(\gamma_{1} \neq \gamma_{0}\right)$ est hors du spectre $\ell^{2}$ de $R^{\prime}$, si et seulement si il existe $\varepsilon=\left(\varepsilon_{i}\right)_{1 \leq i \leq q+1} \in\{ \pm 1\}^{q+1}$ et $\alpha_{0} \in \mathbb{R}$ tels que $\gamma_{1}=\gamma\left(\alpha_{0}\right)$ pour:

$$
\gamma(\alpha)=2 \alpha+\beta_{0}^{\prime}+\sum_{i=1}^{q+1} \frac{-2 \alpha+\left(\ell_{i}-1\right) \beta_{i}^{\prime}+\varepsilon_{i} \sqrt{\left[2 \alpha-\left(\ell_{i}-1\right) \beta_{i}^{\prime}\right]^{2}+4 \ell_{i} \beta_{i}^{\prime 2}}}{2}
$$

avec les conditions:

$$
\begin{aligned}
& \text { i) }\left(\frac{d \gamma}{d \alpha}\right)_{\mid \alpha_{0}}>0 . \\
& \text { ii) } \mathrm{Si}\left(\ell_{i}, \beta_{i}^{\prime}\right)=\left(\ell_{j}, \beta_{j}^{\prime}\right) \text { alors } \varepsilon_{i}=\varepsilon_{j} .
\end{aligned}
$$

Démonstration. Par unicité de l'inverse de $\gamma-R^{\prime}$ et comme $R^{\prime}$ est réel, si $\gamma$ est réel, l'inverse de $\gamma-R^{\prime}$ est à coefficients réels et donc $\alpha$ et les $\eta_{i}(\gamma)$ sont réels. L'expression de $\gamma$ équivaut au a) ci-dessus ([K-R.2; 6]). On a vu que ii) résulte de $a$ ) et $b$ ) (Remarque 2.11), il reste donc à voir que, modulo ii), i) est équivalent à $b$ ).

Pour $\gamma$ réel, positif, grand, alors $\alpha$ est réel positif et les $\eta_{i}$ sont réels positifs et petits donc les $\varepsilon_{i}$ sont tous égaux à 1 . On va considérer un nombre $\gamma^{+}$analogue à $\gamma$ et noter $\alpha^{+}, \eta_{i}^{+}(1 \leq i \leq q+1)$ les nombres correspondants.

Posons $g_{\gamma}^{\prime}=R_{\gamma}^{\prime} * \delta_{s_{0}} \in \ell^{r}\left(S^{\prime}\right)$; on a:

$$
\begin{aligned}
\gamma^{+}\left\langle g_{\gamma^{+}}^{\prime} \mid g_{\gamma}^{\prime}\right\rangle & =\left\langle\gamma^{+} g_{\gamma^{+}}^{\prime} \mid g_{\gamma}^{\prime}\right\rangle=\left\langle R^{\prime} * g_{\gamma^{+}}^{\prime}+\delta_{s_{0}} \mid g_{\gamma}^{\prime}\right\rangle \\
& =\left\langle R^{\prime} * g_{\gamma^{+}}^{\prime} \mid g_{\gamma}^{\prime}\right\rangle+g_{\gamma}^{\prime}\left(s_{0}\right)=\left\langle g_{\gamma^{+}}^{\prime} \mid R^{\prime} * g_{\gamma}^{\prime}\right\rangle+g_{\gamma}^{\prime}\left(s_{0}\right) \\
& =\left\langle g_{\gamma^{+}}^{\prime} \mid \gamma g_{\gamma}^{\prime}-\delta_{s_{0}}\right\rangle+g_{\gamma}^{\prime}\left(s_{0}\right)=\gamma\left\langle g_{\gamma^{+}}^{\prime} \mid g_{\gamma}^{\prime}\right\rangle-g_{\gamma^{+}}^{\prime}\left(s_{0}\right)+g_{\gamma}^{\prime}\left(s_{0}\right) .
\end{aligned}
$$

Pour $\gamma^{+} \neq \gamma$ on obtient $\left\langle g_{\gamma^{+}}^{\prime} \mid g_{\gamma}^{\prime}\right\rangle=-\frac{g_{\gamma^{+}}^{\prime}\left(s_{0}\right)-g_{\gamma}^{\prime}\left(s_{0}\right)}{\gamma^{+}-\gamma}=\frac{\alpha^{+}-\alpha}{\gamma^{+}-\gamma} \times \frac{1}{2 \alpha \alpha^{+}}$. 
Par comparaison avec 2.8 (généralisé de façon à calculer $\left\langle g_{\gamma^{+}}^{\prime} \mid g_{\gamma}^{\prime}\right\rangle$ ), on obtient

$$
\frac{1}{2} \times \frac{\gamma^{+}-\gamma}{\alpha^{+}-\alpha}=1-\sum_{i=1}^{q+1} \frac{\ell_{i} \eta_{i} \eta_{i}^{+}}{1+\ell_{i} \eta_{i} \eta_{i}^{+}} .
$$

Cette expression se généralise par prolongement analytique pour des nombres complexes hors du spectre. On obtient tous les signes possibles pour les $\varepsilon_{i}$ en tournant autour des nombres complexes $\frac{1}{2}\left(\ell_{i}-1\right) \beta_{i}^{\prime}$ $\pm \sqrt{-1} \times \beta_{i}^{\prime} \sqrt{\ell_{i}}$ [si $\left(\ell_{i}, \beta_{i}^{\prime}\right)=\left(\ell_{j}, \beta_{j}^{\prime}\right)$ on doit tourner autour du même nombre complexe, on garde donc $\varepsilon_{i}=\varepsilon_{j}$; si $\left(\ell_{i}, \beta_{i}^{\prime}\right) \neq\left(\ell_{j}, \beta_{j}^{\prime}\right)$ on vérifie que les nombres complexes en question sont différents]. A la limite pour $\gamma$ réel hors du spectre et pour $\varepsilon$ vérifiant ii) on obtient:

$$
\frac{1}{2} \frac{d \gamma}{d \alpha}=1-\sum_{i=1}^{q+1} \frac{\ell_{i}\left|\eta_{i}\right|^{2}}{1+\ell_{i}\left|\eta_{i}\right|^{2}} \text {, d'où le résultat. }
$$

Remarque 2.14. 1) Le corollaire 2.13 ramène la détermination du spectre $\ell^{2}$ à l'étude des variations des fonctions réelles $\gamma(\alpha)$ pour les différents signes des $\varepsilon_{i}$. Cette étude n'est pas bien difficile mais il n'y a pas de résultat très marquant sans hypothèse plus précise sur les $\ell_{i}$ et $\beta_{i}^{\prime}$.

2) Pour $\ell_{i}=1, \forall i$ et $\beta_{0}^{\prime}=0$, on retrouve les résultats de A. Figà-Talamanca et T. Steger pour l'arbre formé des sommets de $S^{\prime}$.

\subsection{Application à des noyaux sur $S$.}

On considère un noyau $R$ à valeurs toutes réelles ou toutes imaginaires pures à sauts de longueur 1 sur $S$ (plus précisément $R(s, t) \neq 0 \Longleftrightarrow d(s, t)=1)$, invariant par un groupe $\Lambda$ transitif sur $S^{\prime}$ et vérifiant la condition $\left(M^{\prime}\right)$ de 2.1. On pose $R^{\prime}=R_{{ }_{S^{\prime}}}$ et on reprend les notations précédentes (voir aussi [K-R.2; 7]. On a:

$$
\beta_{i}^{\prime}=R\left(s_{0}, s_{i}\right) R\left(s_{i}, s_{0}\right) \neq 0 \text { et } \beta_{0}^{\prime}=\sum_{i=1}^{q+1} R\left(s_{0}, s_{i}\right) R\left(s_{i}, s_{0}\right)=\sum_{i=1}^{q+1} \beta_{i}^{\prime} .
$$

Supposons que le groupe $\Lambda$ satisfait à la conditon $(U)$ et que $\ell_{1}{\beta_{1}^{\prime}}^{2}>\sum_{i=2}^{q+1} \ell_{i}{\beta_{i}^{\prime}}^{2}$, alors d'après $2.6 \gamma_{0}=\beta_{0}^{\prime}+\left(\ell_{1}-1\right) \beta_{1}^{\prime}$ n'est pas dans le spectre $\ell^{2}$ de $R^{\prime}$ et $R_{\gamma_{0}}^{\prime}\left(s_{0}, s_{0}\right)=0$. Alors $\alpha\left(\gamma^{2}\right)$ a un pôle en $\gamma= \pm \sqrt{\gamma_{0}}$ et on a des estimations des $\eta_{i}$ en $\gamma= \pm \sqrt{\gamma_{0}}$ :

$$
\eta_{1} \sim \frac{-2 \alpha}{\ell_{1} \beta_{1}^{\prime}} \text { et } \eta_{i} \sim \frac{\beta_{i}^{\prime}}{2 \alpha} \text { pour } 2 \leq i \leq q+1 .
$$


En utilisant [K-R.2; 7.2] $\pm \sqrt{\gamma_{0}}$ n'est pas dans le spectre $\ell^{2}$ de $R$ si $\gamma_{0} \neq 0$. Les relations de [K-R.2; 7.5] se prolongent analytiquement à tout le complémentaire du spectre $\ell^{2}$ de $R$ et on peut les utiliser pour estimer le comportement des fonctions $w$ et $\xi_{\gamma}$ en $\pm \sqrt{\gamma_{0}}$. On obtient les équivalences suivantes quand $\gamma^{2}$ tend vers $\gamma_{0}$.

i) donne $w_{\gamma}\left(s_{1}\right) \sim \frac{-\gamma}{2 \ell_{1}}, w_{\gamma}\left(s_{i}\right) \sim \frac{\gamma}{2} \operatorname{pour} 2 \leq i \leq q+1$ et $w_{\gamma}\left(s_{0}\right)=\frac{\alpha}{\gamma}$.

ii) donne $\xi_{\gamma}\left(s_{0}, s_{1}\right) \sim \frac{-2 \alpha}{\gamma R\left(s_{1}, s_{0}\right)}$ et $\xi_{\gamma}\left(s_{0}, s_{i}\right) \sim \frac{R\left(s_{0}, s_{i}\right)}{\gamma} \neq 0$
$\leq i \leq q+1$ pour $2 \leq i \leq q+1$

et iii) donne $\xi_{\gamma}\left(s_{1}, s_{0}\right) \sim \frac{\gamma}{\ell_{1} R\left(s_{0}, s_{1}\right)}$ et $\xi_{\gamma}\left(s_{i}, s_{0}\right) \sim \frac{\gamma R\left(s_{i}, s_{0}\right)}{2 \alpha}$
pour $2 \leq i \leq q+1$.

En particulier en $\pm \sqrt{\gamma_{0}}$, les fonctions $w_{\gamma}(s)$ pour $s \in S^{\prime \prime}$ n'ont pas de pôle, tandis que pour $s \in S^{\prime}, w_{\gamma}(s)$ a un pôle. De plus tout sommet $s \in S^{\prime}$ a un voisin $s_{1}$ (unique si $\gamma_{0} \neq 0$ ) dans $S^{\prime \prime}$ tel que $\xi_{\gamma}\left(s, s_{1}\right)$ a un pôle tandis que pour $s \in S^{\prime}, s_{2} \in \Sigma(s, 1), \quad \xi_{\gamma}\left(s_{2}, s\right)$ n'a jamais de pôle. On peut en fait calculer explicitement $R_{ \pm \sqrt{\gamma_{0}}}(s, t) \quad \forall s, t \in S$, si $\gamma_{0} \neq 0$.

On notera la différence de comportement avec ce que l'on a observé au $\S 1$. En particulier si $\gamma_{0}=0,0$ est dans le spectre $\ell^{2}$ de $R$ mais pas dans celui de $R^{\prime}=R_{{ }_{S^{\prime}}}^{2}$.

\section{REFERENCES}

[A] K. Аомото, Spectral theory on a free group and algebraic curves, J. Fac. Sci., Univ. Tokyo, 31 (1984), pp. 297-317.

[B.K] F. BouAZIZ-KelLIL, Représentations sphériques des groupes agissant transitivement sur un arbre semi-homogène, Bull. Soc. Math. France, 116 (1988), pp. 255-278.

[C-C] J. M. Cohen - F. Colonna, Eigenfunctions of the laplacien on homogeneous tree, Contemp. Math., 206 (1997), pp. 121-124.

[Ft-S] A. Figà-Talamanca - T. Steger, Harmonic analysis for anisotropic random walks on homogeneous trees, Memoirs of AMS, 531 (1994).

[K-R.1] F. Kellil - G. Rousseau, Généralisation d'un théorème de Haagerup, Studia Math., 168 (3) (2005), pp. 217-227.

[K-R.2] F. Kellil - G. Rousseau, Transformation de Poisson sur un arbre localement fini, Ann. Math. Blaise Pascal, 12 (2005), pp. 91-116.

[St] T. STEGER, Harmonic analysis for an anisotropic random walk on a homogeneous tree, Thesis, Washington Univ., St. Louis, 1985.

Manoscritto pervenuto in redazione il 20 luglio 2006. 